Musées, Patrimoine et Culture scientifiques et techniques

$146 \mid 2013$

mars-avril 2013

\title{
Les relations adolescents-musées : comparaison France/États-Unis
}

\section{Caroline Darcq}

\section{OpenEdition \\ Journals}

Édition électronique

URL : http://journals.openedition.org/ocim/1215

DOI : 10.4000/ocim. 1215

ISSN : 2108-646X

\section{Éditeur}

OCIM

Édition imprimée

Date de publication : 1 mars 2013

Pagination : $29-36$

ISSN : 0994-1908

Référence électronique

Caroline Darcq, «Les relations adolescents-musées : comparaison France/États-Unis », La Lettre de I'OCIM [En ligne], 146 | 2013, mis en ligne le 01 mars 2015, consulté le 10 décembre 2020. URL : http:// journals.openedition.org/ocim/1215; DOI : https://doi.org/10.4000/ocim.1215 


\section{Les relations adolescents-musées : comparaison France/États-Unis}

\section{Caroline Darcq *}

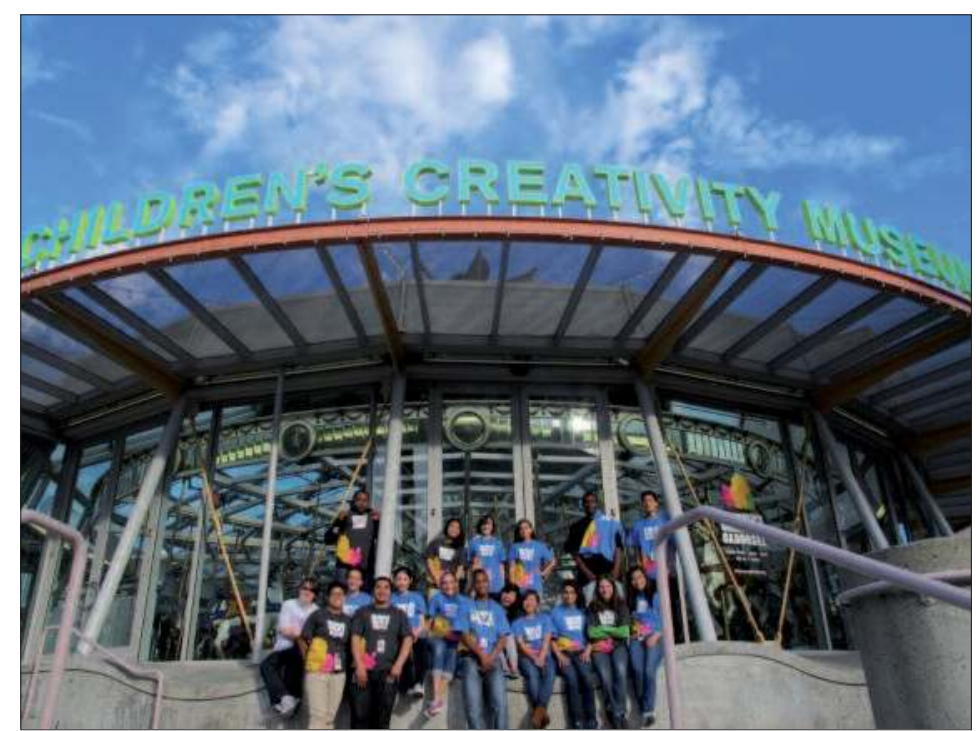

L'équipe des C.I.T.Y. Guides mise en place au muséum d'Histoire naturelle de San Francisco. ๑) Aimée Espiritu/Children's Creativity Museum, 2012

* Caroline Darcq est titulaire d'un Master 2 Médiation de la Culture et du Patrimoine de l'université d'Avignon et des Pays du Vaucluse caroline.darcq@gmail.com
À travers un état des lieux des relations adolescents-musées en France et aux États-Unis, l'auteur analyse le rôle et la spécificité de l'offre éducative diffusée par des dispositifs muséaux réservés aux adolescents en visite libre, montre que les efforts déployés ne sont pas toujours couronnés de succès et qu'au-delà du cadre de l'institution muséale, se pose la question de la place concédée à la classe d'âge adolescente dans la société.

En France et aux États-Unis, les adolescents sont peu présents dans les institutions muséales. La principale raison donnée à leur refus de s'y rendre est la représentation négative du musée qu'ils en ont : il serait passéiste et ennuyeux. Les adolescents l'associent à une boring armour (armure de l'ennui) que renforce en grande partie la pression sociale de leurs pairs (O’Riain, 1997). Il est ainsi plutôt malvenu pour un adolescent de déclarer à ses amis que les musées sont intéressants.

Les adolescents s'entendent pour dire que les musées ne sont pas pour eux mais plutôt pour les adultes et les enfants. Pour les adolescents, la visite du musée est rattachée à l'univers parental. De plus, en France, les activités proposées aux adolescents par les institutions muséales sont souvent similaires à celles proposées aux enfants.

\section{Les adolescents et l'adolescence en France et aux États-Unis}

On admet depuis assez longtemps l'existence d'un groupe social reconnu comme distinct de l'enfance et 
de l'adulte par la spécificité de son âge, de sa place sociale, de ses comportements, de ses goûts et de son mode de fonctionnement psychique. Cependant, les mutations sociales qui renouvellent la définition de la classe d'âge adolescente rendent difficile son appréhension. L'adolescence est une construction historique rigoureusement associée à l'allongement de la formation scolaire et à la démocratisation de l'éducation. Aujourd'hui, la culture adolescente est reconnue par l'uniformisation relative des pratiques culturelles et des modes de vie. La culture adolescente est devenue interclassiste, elle s'est massifiée. Le fossé entre la culture adolescente et la culture légitime, associée aux normes imposées par les institutions telles que le musée, se creuse. La majorité des institutions muséales françaises s'intéressant à ce public conçoit l'adolescence comme une catégorie uniforme, sans aspérité, d'individus âgés de 11 à 18 ans.

Il semblerait cependant que la classe d'âge adolescente se caractérise avant tout par son hétérogénéité. Les regards portés par les institutions muséales sur l'adolescent tendent ainsi à sous-estimer la diversité de cette population et les tensions internes qui l'animent. François de Singly (2006) distingue de l'adolescence, l'adonaissance. Les comportements des 11-14 ans et des 15-18 ans étant très différents, il souligne le double mécanisme de distinction à la fois intergénérationnelle et intra-générationnelle, entretenu par les adolescents. Le processus d'individualisation débuterait ainsi au début de l'adonaissance et s'achèverait à la fin de l'adolescence à travers deux mouvements de projection distincts créant deux classes d'âges distinctes. L'existence de ces deux classes d'âge semble davantage prise en compte par les institutions muséales américaines qui proposent des médiations différentes aux adolescents inscrits à la Middle School ou Junior High School (correspondant en France au collège) et ceux inscrits à la High School (lycée).

Aux États-Unis et en France, les institutions muséales considèrent le public adolescent comme difficile à saisir. Si la culture adolescente est souvent considérée comme étant à l'opposé de la culture légitime transmise par les musées, il est important de souligner le fait que l'adolescent est notamment défini par une forte curiosité et une réelle soif de connaissances. L'adolescence est la période privilégiée des pratiques de loisirs et des apprentissages.

En France, la faible prise en compte des adolescents et de leurs attentes au sein des institutions muséales renvoie plus largement à une faible intégration sociale de ces derniers. Les adolescents français rencontrent de nombreuses difficultés pour atteindre le statut d'adulte. Une étude internationale traitant des regards des jeunes sur leur avenir (Stellinger, 2008), montre que les adolescents français comptent parmi les plus pessimistes. Alors que plus de la moitié des jeunes américains pensent que leur avenir est prometteur, moins d'un quart des jeunes français en sont convaincus. Cette différence peut s'expliquer par le peu de confiance que la société française accorde à ses adolescents. Ce pessimisme s'inscrit dans le temps et dans une dimension intergénérationnelle, il paralyse les jeunes français et casse leurs ambitions professionnelles. Le développement de la culture adolescente entraîne un repli sur les groupes de pairs et participe à l'affaiblissement de valeurs collectives et intergénérationnelles. Aux États-Unis, il n'existe pas de consensus dans la manière de considérer les adolescents mais la société américaine s'efforce de leur confier une place plus importante. Les institutions muséales américaines sont notamment plus nombreuses à proposer des médiations spécifiques aux adolescents.

Mais un autre trait complique ce tableau : l'instauration du jeunisme. La culture adolescente a acquis une réelle dimension consumériste même si c'est ce qui incite les musées à considérer les adolescents comme des non publics (Jacobi et Luckerhoff, 2012). Cependant il ne faut pas oublier que la consommation adolescente possède une réelle dimension culturelle. La culture adolescente ne conteste plus le modèle de société mais au contraire elle y participe pleinement (Galland, 2009). Les adolescents sont non seulement des gros consommateurs mais aussi des modèles pour les générations plus âgées.

\section{Les offres muséales destinées aux adolescents}

En France à partir des années 1990 et aux États-Unis à partir des années 1970, les institutions muséales ont

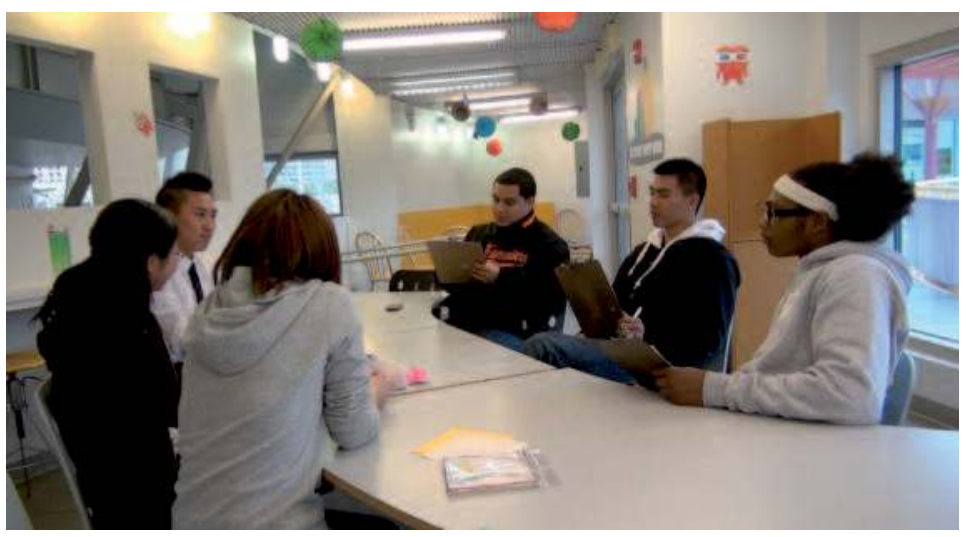

Des C.I.T.Y. Guides mènent un entretien pour recruter leurs futurs collègues. (c) Caroline Darcq/Children's Creativity Museum, 2012 
développé des médiations dédiées aux adolescents en visite libre. Ces différentes tentatives n'ont pas toujours été efficientes. Ceci semble pouvoir s'expliquer par le manque de spécificité de ces offres qui ne s'adaptent pas à la classe d'âge adolescente. La majorité des offres pour adolescents proposées par les musées français rencontrent des difficultés pour trouver leur public hors temps scolaire. Cela tient sans doute au cadre trop rigide des ateliers. Les adolescents doivent y suivre les instructions des médiateurs et réaliser en groupe les mêmes activités au même rythme et en même temps. Les horaires étant imposés, ils ne sont pas invités à décider de l'heure et de la durée de leur visite. De plus, ces ateliers sont souvent payants ce qui oblige les adolescents à rester majoritairement dépendants de leurs parents. Même si le manque d'intérêt des adolescents vis-à-vis des musées est un obstacle plus difficile à surmonter que le prix, la gratuité est l'un des facteurs facilitant la visite des adolescents en autonomie. Des cartes, offrant un accès privilégié et illimité aux différentes collections et expositions, aux visites guidées ainsi qu'aux ateliers et autres manifestations ont ainsi été conçues par les institutions muséales. En France, ces cartes semblent toutefois cibler davantage les jeunes adultes de 18 à 26 ans. Les adolescents ne forment pas le public cible de ces dispositifs d'autant plus que de nombreux musées sont gratuits pour les moins de 18 ans. Certains musées américains comme The Metropolitan of Art de New York, proposent des teen pass offrant la gratuité et un accès privilégié à l'institution, ces cartes sont souvent exclusivement reversées aux high school students (lycéens).

Alors que les musées français se contentent majoritairement de proposer des ateliers inspirés de ceux pour les plus jeunes enfants, des projets spécifiques impliquant les adolescents dans le fonctionnement des institutions muséales se sont développés aux ÉtatsUnis (teen programs). Aujourd'hui, de nombreux musées américains proposent aux adolescents de travailler, de manière rémunérée ou non, pour les institutions. À titre d'exemple, le Children's Creativity Museum de San Francisco, a mis en place le C.I.T.Y. (Creative Inspiration Through Youth) teen program, des lycéens travaillent hors temps scolaire et de manière rémunérée. À travers ce programme, des lycéens sont embauchés chaque année en tant que médiateurs. Les C.I.T.Y. Guides transforment le visage du musée et permettent de rajeunir l'image de l'institution. Ce dispositif intègre les adolescents dans l'équipe du musée durant une période d'un an au minimum. Les adolescents peuvent intégrer le programme dès l'âge de 15 ans et poursuivre cette expérience jusqu'à 18 ans. Durant la dernière année de ce programme, ils peuvent bénéficier de promotions et ainsi devenir chef d'équipe, régisseur ou agent d'accueil. Ils ont un rôle très important dans le fonctionnement du musée. Ils sont aussi nombreux que le reste de l'équipe et ils travaillent en étroite collaboration avec eux. Parallèlement à leurs heures de travail, les adolescents sont invités à participer à différents ateliers portant sur des thématiques artistiques et muséales. En intégrant ce programme, les adolescents reçoivent une carte, qui leur offre un accès gratuit à l'ensemble des institutions muséales et à certains événements culturels de la ville. En offrant aux adolescents une place privilégiée, ces programmes reposent sur une approche behind the scene qui offre aux adolescents un accès aux coulisses du musée. Ces programmes développent des relations sur le long terme entre représentants de la culture adolescente et de la culture légitime.

Pour conquérir ce public, certaines institutions muséales ont offert une véritable place à l'adolescent, à ses modes de vie et ses habits. Les offres culturelles à destination des adolescents ont proposé des modalités de visite souples et flexibles. Ainsi, le Contemporary Jewish Museum de San Francisco propose des teen opening, des ouvertures spécifiques organisées en dehors des horaires classiques du musée.

Sans proposer des horaires spécifiques, les musées français ciblent le temps libre des adolescents : les vacances scolaires, le mercredi après-midi et le weekend. Le Centre Pompidou a créé le Studio 13/16, un espace séparé du reste de l'institution et spécialement

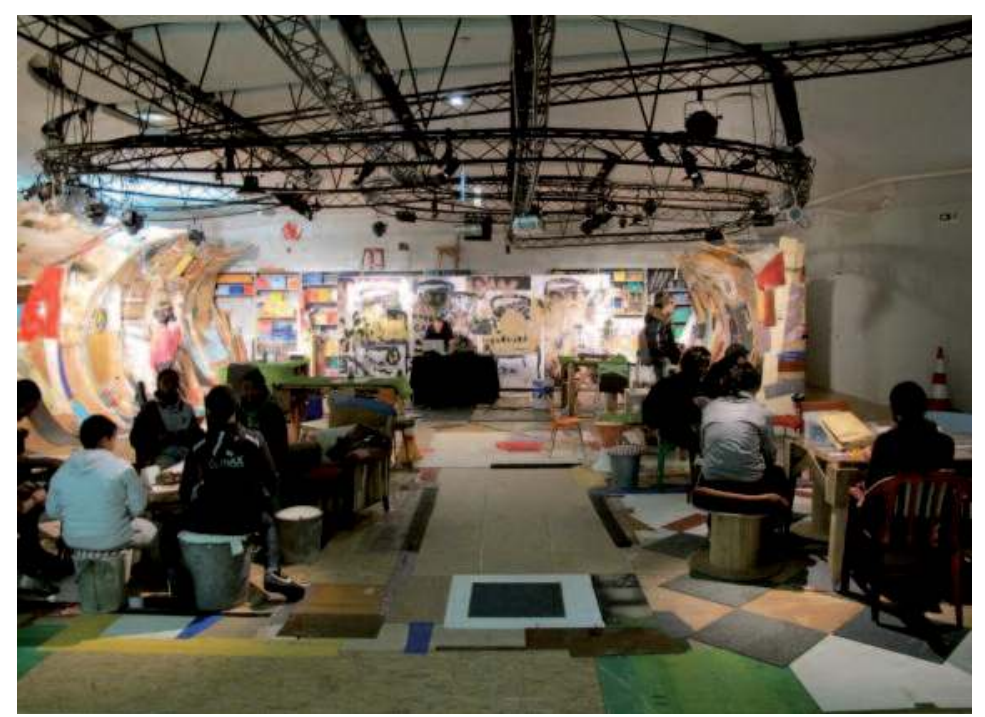

L'exposition Green Attitude au Studio 13/16 du Centre Pompidou ๑) Hervé Véronèse/Centre Pompidou, 2011 
dédié aux adolescents. Ce projet a été fondé sur la conviction que l'adolescence est la période de la vie où se cristallise la relation à l'art et à l'institution culturelle. Ainsi, la création d'une relation privilégiée entre le musée et les adolescents constitue une démarche irremplaçable pour former les visiteurs de demain. Situé au cœur du Centre Pompidou, il est un lieu aisément accessible. Les modalités d'accueil facilitent la venue des adolescents, ils n'ont pas besoin de réserver et l'accès y est gratuit. Cet espace s'adresse aux adolescents à travers différentes thématiques renouvelées tous les trois mois environ. Ce lieu modulable propose un programme constitué de rencontres avec des artistes, d'expériences inédites autour d'œuvres interactives, de soirées et week-ends festifs. Les ateliers proposés sont résolument pluridisciplinaires et couvrent les différents domaines de la création contemporaine : arts visuels, danse, musique, cinéma, mais aussi le champ des loisirs culturels comme les jeux et les clips vidéo, les différents modes de diffusion numériques ou le design. Afin de s'adapter à l'exigence de flexibilité des adolescents, aucun atelier n'est organisé à horaire fixe. On leur propose de découvrir l'espace à leur rythme, de l'expérimenter seuls ou au sein d'un groupe de pairs.

Le Centre Pompidou souhaite favoriser la visite en autonomie, offrir un lieu informel où se retrouver entre copains, en dehors de leur scolarité. Mathieu Lehanneur, le designer de l'espace, souhaite que les adolescents du Studio puissent s'y retrouver, y draguer, se surprendre eux-mêmes et pourquoi pas s'endormir. Cet encadrement non rigide permet aux adolescents de se retrouver entre eux, de se sentir à l'aise et de trouver leur place au sein de l'institution.

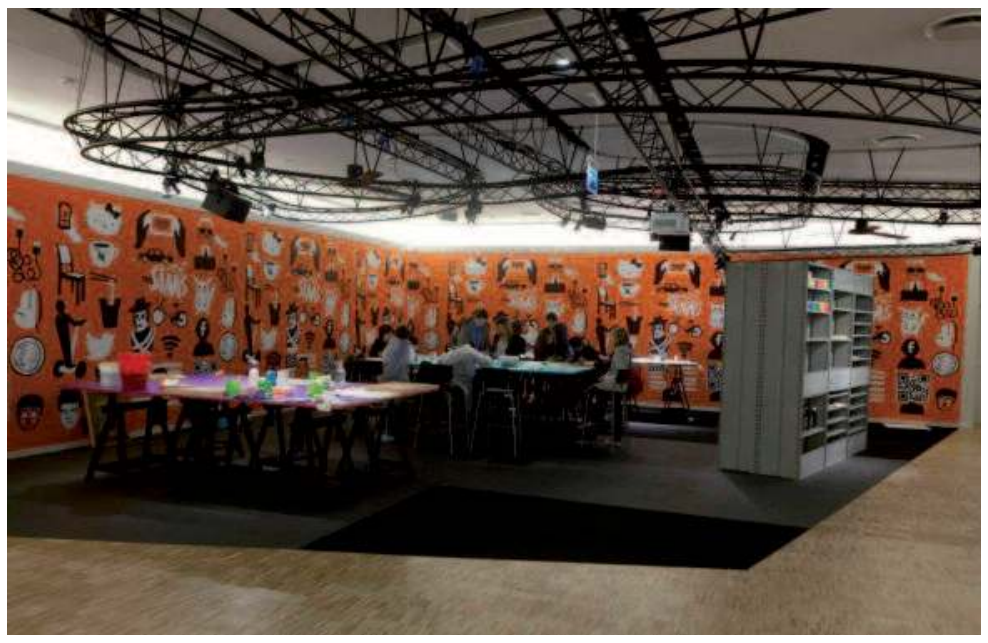

L'exposition Serial Printer au Studio 13/16: des thèmes et des codes issus de la culture adolescente. ๑) Hervé Véronèse/Centre Pompidou, 2012
Parallèlement, il ne faut pas oublier que les adolescents se caractérisent également par une dépendance à l'égard de deux instances de socialisation : la famille et l'école. L'autonomie dans la gestion de leurs déplacements, dont les adolescents jouissent de plus en plus tôt, est limitée. Le temps passé avec le groupe de pairs, hors des influences familiale et scolaire, reste relativement marginal. Afin d'attirer ces publics, et sans oublier de favoriser leur individualisation, les institutions muséales s'adaptent à différentes contraintes imposées par ces deux instances. Il s'agit tout de même de convaincre les adolescents eux-mêmes et non pas uniquement les adultes qui les entourent. Certains musées s'efforcent de séduire les adolescents en intégrant des thèmes et des codes issus de la culture adolescente. Les nouveaux movens de communication, dont le développement est très important chez les adolescents, jouent un rôle important dans la diffusion de la culture adolescente. Ces modes de communication convoquent de nouveaux codes que les institutions muséales doivent intégrer pour se faire une place sur le marché adolescent. Des institutions muséales s'appuient sur les réseaux sociaux pour distiller des informations vers les utilisateurs. Cette nouvelle forme de communication cherche à encourager la participation à travers la publication de commentaires. Il s'agit de former une communauté d'adolescents-visiteurs. Sur ces lieux virtuels, le contrôle parental n'est que très rarement exercé, les adolescents y développent leur autonomie. Pour attirer ces adolescents expérimentant le virtuel, les musées proposent des espaces en ligne dédiés à la classe d'âge adolescente afin qu'elle puisse poursuivre sa visite hors les murs. Par ailleurs, l'apparence étant au cœur des préoccupations adolescentes actuelles, la distribution d'objets dérivés, comme véritables accessoires de modes, peut également permettre de redorer l'image de l'institution. Distribués dans les institutions muséales ou lors de missions de street marketing, ils peuvent permettre de convaincre les adolescents de se rendre au musée.

À travers ces dispositifs, il ne s'agit pas uniquement d'intégrer la culture adolescente au monde muséal, l'exploitation de ces thématiques a plutôt pour objectif d'attirer les adolescents afin d'élever leur niveau de curiosité, de former les visiteurs de demain et les futurs acteurs de la société.

L'intérêt des musées pour les adolescents et les efforts actuellement déployés pour rejoindre ces publics ne reposent pas uniquement sur une approche marketing et sur le besoin des musées d'accroître leur nombre de visiteurs. Des objectifs éducatifs et sociaux sont 


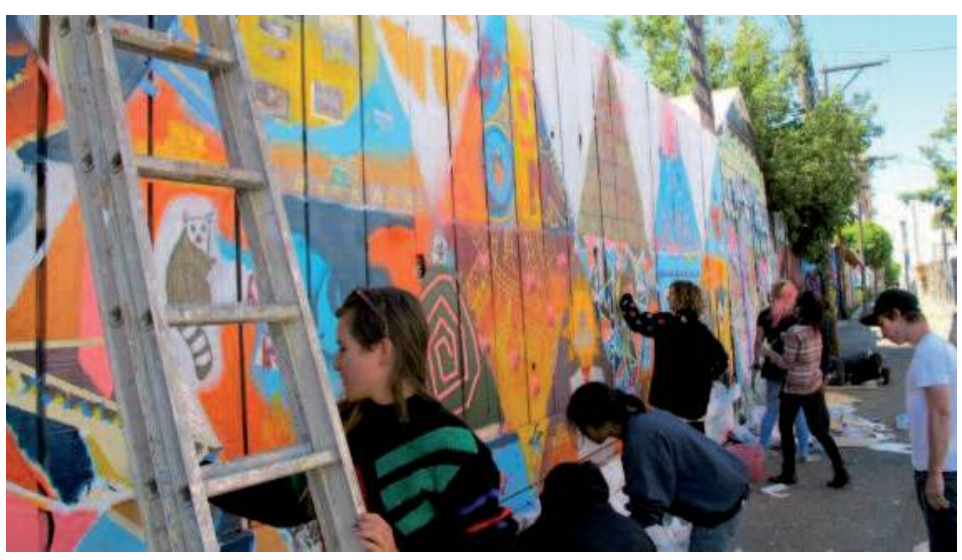

Les C.I.T.Y. Guides participent à un atelier street art avec les artistes Callen Zimmerman et Whitney Sharpe. ๑ Aimée Espiritu/quartier Mission de San Francisco, 2012

En France, les médiations dédiées aux adolescents en visite libre forment une autre source d'acculturation que l'appareil scolaire, elles appartiennent à l'éducation non formelle construite en opposition à l'enseignement académique (Jacobi, 2001). Les ateliers ont pour objectif d'être propices à la découverte d'autres façons de penser, de vivre, d'imaginer le monde. La participation à ces ateliers sollicite d'autres références, d'autres critères, d'autres intelligences et d'autres attitudes que ceux de l'école. Certains adolescents considèrent ces ateliers plus intéressants car ils se sentent davantage concernés par les savoirs diffusés. L'absence d'évaluation permet aux adolescents de se sentir plus à l'aise, d'exposer leurs points de vue sans avoir peur de donner un mauvais résultat et d'être sanctionné. Cette liberté permet notamment aux adolescents d'acquérir une autonomie et de développer leur créativité. Ces espaces semblent privilégier le développement d'une pensée divergente différente de la pensée convergente qui domine dans le secteur scolaire. Le déroulement de ces ateliers n'est pas identique à celui d'un cours au collège ou au lycée. Aller à la rencontre des objets muséaux implique des approches pédagogiques qui sont souvent complémentaires à celles utilisées à l'école. La rencontre avec les œuvres est personnelle, cependant le partage de cette rencontre entre l'ensemble du groupe de pairs est essentiel pour la signifier, voire la valoriser. Les moments conviviaux sont ainsi encouragés et permettent un libre échange entre les participants. Ces médiations favorisent un déroulement informel et conversationnel où les travaux de groupe et les échanges sont mis en valeur. Ces ateliers se rapprochent du modèle politique d'éducation américain qui propose aux adolescents d'expérimenter le monde.
Aux États-Unis, les médiations spécifiques des adolescents apportent d'autres compétences complémentaires de celles apportées à l'école, elles permettent à l'adolescent de s'épanouir davantage en tant que futur travailleur. Les adolescents ont conscience très tôt de la concurrence qui s'exerce sur le marché du travail. Ceci entraîne une scolarisation des temps sociaux, le modèle de conduite est en partie acquis hors de l'école.

À l'adolescence, le temps libre conquis sur le temps contraint nourrit le sentiment de liberté. Aux ÉtatsUnis, même si les journées scolaires sont plus courtes, les adolescents s'impliquent dans de nombreuses activités qui réduisent considérablement leur temps libre. Afin de pouvoir intégrer les meilleures universités voire même les meilleurs lycées, les jeunes américains sont conditionnés dès le plus jeune âge à réussir dans tous les domaines. Cette pression est moins forte en France où on assiste également au maintien des normes scolaires à l'extérieur de l'école. Les activités de loisirs doivent être envisagées comme des moments de liberté et permettre aux adolescents d'être autonomes à travers la maîtrise de leur temps. Or, le loisir des adolescents est souvent pensé comme occupationnel : on leur impose des activités pour qu'ils ne gaspillent pas leur temps à être inactifs. Les activités artistiques et culturelles proposées aux élèves hors temps scolaires correspondent aux valeurs véhiculées par l'école. Les élèves les plus favorisés culturellement et socialement sont ainsi incités à participer à des activités muséales (Zaffran, 2011). Ces obligations parentales peuvent être assimilées par les adolescents à une contrainte et ainsi encourager leur représentation négative de l'institution muséale. En France, les adolescents participant aux ateliers muséaux proviennent majoritairement de milieux favorisés. Sans y être forcément contraints et ayant intégré des pratiques de visite dans le cadre familial, ils se rendent au musée à leur guise. Afin de combattre ce déterminisme, les responsables des médiations américaines, en relation avec les acteurs de l'école, s'efforcent d'établir une véritable mixité sociale dans les équipes adolescentes. Des adolescents aux parcours divers peuvent ainsi bénéficier ensemble des nombreux apports éducatifs diffusés par ces programmes.

\section{Empowerment : I'enjeu majeur des relations adolescents-musées}

L'accès à la conscience de soi est le premier enjeu de l'adolescence. Cette conscience de soi est découverte progressivement à travers différentes expériences. Ces 


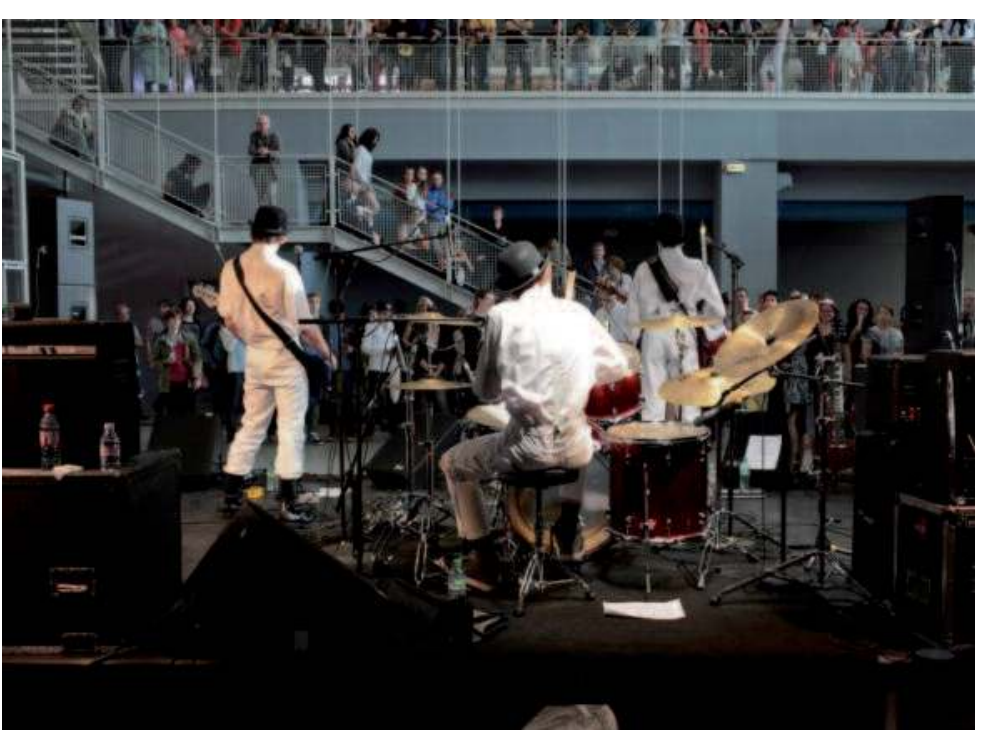

Des animations propres à assurer la venue des adolescents au musée : I'événement On Air au Studio 13/16

du Centre Pompidou.

(c) Hervé Véronèse/Centre Pompidou, 2012

médiations doivent permettre aux adolescents de développer leur processus d'individualisation en s'éloignant du cadre familial et en se socialisant. Il s'agit de permettre aux adolescents qui considèrent fréquemment que le musée s'adresse uniquement aux adultes, de s'approprier l'espace muséal de manière personnelle et dans un cadre générationnel, loin de leurs parents. À travers le développement de cette autonomie, les concepteurs de ces espaces ont pour objectif de donner des moyens aux adolescents d'agir sur leur environnement.

Les teen programs américains permettent d'offrir aux institutions de nouvelles perspectives en donnant la parole aux adolescents et en intégrant leurs conseils dans les lignes directrices. Ces initiatives visent l'empowerment, soit l'acquisition de la capacité d'agir. À travers ce processus basé sur l'échange, on cherche à rendre les adolescents autonomes et volontaires, acteurs de leur vie et de la société. L'absence de traduction française du mot empowerment est le reflet des différences culturelles énoncées précédemment. Ce mot anglais ne peut pas être traduit par le mot responsabilisation qui renvoie à une forme d'obligation et à une certaine hiérarchie. À travers l'empowerment, il s'agit d'intégrer des adolescents dans la société active en les invitant à jouer des rôles dans un musée qui leur permet de le faire. Les adolescents devenant membres d'une équipe intergénérationnelle possèdent un droit de parole, une influence sur les politiques et les projets des institutions muséales. Ces programmes permettent de casser réellement les barrières entre culture adolescente et culture muséale.

En France, aucun véritable rôle ne leur est confié, Ils participent à des ateliers mais n'en sont pas moteurs. On offre une place aux adolescents au sein des publics de l'institution mais pas les moyens d'agir sur sa politique. Dans la majorité des activités proposées aux adolescents dans les institutions muséales françaises, les consignes trop présentes nuisent à l'autonomie des participants. Si les adolescents ont l'impression de devoir se contenter de suivre des instructions, ils considéreront les adultes présents dans l'espace comme des professeurs et l'atelier comme un cours durant lequel ils n'ont pas le droit de s'exprimer librement. Le fait d'avoir un certain rôle au sein des institutions muséales, permet aux adolescents américains de se libérer des cadres protecteurs pour se construire et trouver leur place dans la société.

Cette différence reflète la place concédée à la classe d'âge adolescente dans ces deux sociétés. Les jeunes américains sont encouragés très tôt à avoir des responsabilités et un rôle actif dans la société. L'effet attendu est de réduire la distance entre adolescents et adultes et de favoriser le sentiment qu'il est possible d'avoir un impact concret sur le monde. Les adolescents s'impliquent ainsi dans différents projets volontaires ou non, s'expriment et agissent en tant qu'individu. L'âge de travail légal et les conditions pour travailler sont plus souples aux États-Unis. De plus, en France, on incite peu les adolescents à s'engager socialement. En France, la faible intégration des adolescents français dans la société les place hors jeu socialement.

\section{Culture adolescente versus culture muséale?}

Le véritable défi est de déconstruire cette dichotomie créant des barrières artificielles entre adolescents et institutions muséales. Il s'agit de juxtaposer la culture adolescente et la culture légitime, de casser l'image d'une culture populaire, d'un côté, et noble, de l'autre. Afin de renouveler les relations adolescents-musées, certaines institutions muséales proposent des dispositifs de médiation exclusivement réservés aux adolescents. Le risque encouru est de concevoir des médiations non adaptées et d'enfermer ainsi l'adolescent dans une catégorie superficielle (Bruno, 2000).

L'institution muséale française pourrait s'organiser afin d'encourager les adolescents à inventer et entreprendre afin qu'ils puissent la faire évoluer. Afin de participer au processus d'individualisation des adolescents, les 
institutions muséales gagneraient à proposer une offre réellement spécifique des groupes de pairs adolescents. Les pratiques culturelles des adolescents peuvent ainsi leur permettre de se distinguer de leurs parents et de leur enfance, de s'identifier à une classe d'âge et de devenir membre d'une génération qui joue un rôle dans la société.

Les institutions muséales américaines proposent une offre s'adaptant aux besoins et attentes spécifiques des adolescents. Le risque cependant encouru par les teen programs est d'amener trop vite l'adolescent vers le statut d'adulte. Dans quelle mesure les institutions françaises doivent-elles être influencées par le modèle américain ? Les différences culturelles entre les deux pays empêchent la naissance de teen programs en France. Il semble pourtant important pour renouveler les relations adolescents-musées de donner un droit de parole aux adolescents et de ne plus se baser uniquement sur des analyses a priori enfermant l'adolescent dans une catégorie superficielle.

\section{Bibliographie}

Baum, L. In their own words : voices of teens in museums, The Journal of Museum Education, vol. 25, n³, 2000, pp. 9-14.

Bruno, P. Existe-t-il une culture adolescente? Paris : In press Éditions, 2000.

De Singly, F. Les Adonaissants. Paris : Armand Colin, 2006.

Fize, M. Les adolescents. Paris : Éditions Le Cavalier Bleu, 2009.

Galland, O. Les jeunes français ont-ils raison d'avoir peur? Paris : Armand Colin, 2009

Jacobi, D. Les dispositifs non scolaires d'acculturation : introduction à l'analyse de l'éducation non formelle, Pour, n¹65, 2001, pp. 187-192.

Jacobi, D. et Luckerhoff, J. Looking for non-publics. Presses de l'université du Québec, 2012.
Lemerise, T. The role and the place of Adolescents in museums : yesterday and today, Museum Management and Curatorship, vol. 14, n4, 1995, pp. $393-408$

Meuret, D. Gouverner l'école : une comparaison France/États-Unis. Paris : PUF, 2007

O'Rian, H. Chinks in the «Boring » armour, GEM News, Group for Education in museums, ${ }^{\circ} 65,1997$, pp. 11-15

Stellinger, A. et Wintrebert, R. Les jeunesses face à leur avenir : une enquête internationale. Université du Michigan : Édition Fondation pour l'innovation politique, 2008.

Zaffran, J. Le Temps de l'adolescence : entre contrainte et liberté. Rennes : Presses de l'université de Rennes, 2011. 